\title{
Evaluación de la calidad tecnológica, nutricional y sensorial de barras de cereal con quinoa
}

\author{
Steffolani, M. E., Bustos, M. C., Ferreyra, M. E. y León, A. E.
}

\begin{abstract}
RESUMEN
La semilla de quinoa (Chenopodium quinoa Willd) se caracteriza por presentar una composición balanceada; es rica en proteínas, fibra, vitaminas, minerales y antioxidantes, por lo que puede ser incorporada en la formulación de barras de cereal a fin de incrementar el valor nutricional de la alimentación diaria. El objetivo de este trabajo fue estudiar el efecto de la incorporación de semillas de quinoa en la calidad tecnológica, nutricional y sensorial de barras de cereal. Se elaboraron y analizaron tres muestras de barras de cereal, con diferentes porcentajes de sustitución de arroz crocante por semillas de quinoa y un control (sin quinoa). Se observó un incremento de peso de las barras de cereal, a medida que se aumentó el nivel de sustitución y una disminución en el largo de éstas. La dureza de las barras fue afectada, obteniéndose barras más blandas cuando tenían quinoa en la formulación. La incorporación de quinoa generó un aumento en el contenido de minerales, proteínas y fibra alimentaria del producto final. Además, las barras de cereal con quinoa presentaron una digestibilidad más lenta del almidón. A los consumidores les resultó agradable el sabor de las barras con quinoa y también tuvieron una buena aceptabilidad global.
\end{abstract}

Palabras clave: quinoa, cultivo andino, barra de cereal, almidón digerible, aceptabilidad.

Steffolani, M. E., Bustos, M. C., Ferreyra, M. E. y León, A. E., 2017. Evaluation of technological, nutritional and sensory quality of cereal bars with quinoa. Agriscientia 34 (II): 33-43

\section{SUMMARY}

Quinoa seed (Chenopodium quinoa Willd) is characterized by a balanced composition, rich in proteins, fiber, vitamins, minerals and antioxidants; so, it can be incorporated into the formulation of cereal bars in order to increase the nutritional value of the daily diet. Therefore, the objective of this work was to study the effect of quinoa seed incorporation on the technological, nutritional and sensory quality of cereal bars. Three samples of cereal bars were prepared with different percentages of substitution of crispy rice with quinoa seeds and a control (without addition of quinoa). An increase in weight and a decrease in 
the length of the cereal bars were observed, as the level of quinoa increased in the formulation. The hardness of the bars was affected, obtaining softer bars when they had quinoa in their formulation. The incorporation of quinoa generated an increase in the content of minerals, proteins and dietary fiber of the final product. In addition, the quinoa cereal bars showed a slower starch digestibility. Consumers liked the taste of quinoa bars and they also had good overall acceptability.

Key words: quinoa, Andean crops, cereal bars, digestible starch, sensory acceptability.

M. E. Steffolani y A. E. León: Instituto de Ciencia y Tecnología de los Alimentos Córdoba (ICYTAC), CONICET-UNC. Facultad de Ciencias Agropecuarias, Universidad Nacional de Córdoba, CC 509, 5000. Córdoba, Argentina. M. C. Bustos: Instituto de Ciencia y ecnología de los Alimentos de Córdoba (ICYTAC), CONICETUNC. M. E. Ferreyra: Georgalos HNOS SAICA. Correspondencia a: eusteffolani@agro.unc.edu.ar

\section{INTRODUCCIÓN}

La domesticación y mejora de algunas especies vegetales, más precisamente de cereales, ha llevado al abandono de muchos cultivos andinos altamente nutritivos como la quinoa, semilla nativa que se cultiva y crece en la región andina. La quinoa, además de tener mayor valor nutricional respecto de los cereales tradicionales, ha sido asociada a la disminución del riesgo de padecer algunas enfermedades crónicas relacionadas con la dieta por su alto contenido de minerales, vitaminas, ácidos grasos y antioxidantes (Repo-Carrasco, Cortez, Quispe y Ramos, 2006; Vega-Gálvez, Miranda y Vergara, 2010). Por iniciativa del gobierno boliviano, la Organización de las Naciones Unidas para la Alimentación y la Agricultura (FAO) declaró el 2013 como el Año Internacional de la Quinoa. En este sentido, los esfuerzos se han centrado en promover la semilla a fin de potenciar los cultivos tradicionales u olvidados como medio para combatir el hambre y fomentar una alimentación saludable.

La quinoa crece principalmente en zonas con condiciones climáticas y ecológicas desfavorables para la mayoría de los cultivos, debido a que se adapta a un amplio rango de condiciones ambientales; además, tiene una gran resistencia a las heladas, salinidad del suelo, sequías y enfermedades, pudiendo tolerar en sus suelos un amplio rango de $\mathrm{pH}$ (entre 4,8 y 9,5) (Jacobsen, Mujica y Jensen, 2003).

El almidón es el principal carbohidrato en la quinoa (aproximadamente entre el 60 y el $70 \%$ en base seca), como en todos los cereales, pero se encuentra principalmente en el perisperma. Los gránulos de almidón son poligonales, irregulares, angulares y pequeños, con un diámetro de entre 1,0 y $3,5 \mathrm{~mm}$. El almidón de la quinoa gelatiniza en un rango de entre 50 y $60^{\circ} \mathrm{C}$ y las temperaturas de retrogradación de la amilopectina son más bajas (entre 45 y $55^{\circ} \mathrm{C}$ ) que en la mayoría de los cereales (Steffolani, Leon y Perez, 2013). Además, el contenido de amilosa es más bajo (entre 9 y $10 \%$ en promedio). En comparación con los cereales, el contenido total de proteínas (12 $23 \%$ ) es mayor que en la cebada, arroz o maíz, y es comparable a la de trigo (Abugoch, Romero, Tapia, Silva y Rivera, 2008). Sin embargo, la importancia de las proteínas de la quinoa se debe principalmente a su calidad y no a su cantidad. Según los requerimientos sugeridos por la WHO (1985) para niños mayores de 5 años, algunas variedades de este cultivo no poseen aminoácidos limitantes y el contenido de lisina (aminoácido limitante en la mayoría de los cereales) se encuentra duplicado con respecto a otros cereales, como el trigo (Steffolani et al., 2015). Algunos estudios han demostrado que la quinoa es una buena fuente de fibra dietética, con un rango de 1,1 a 16,3\%, por lo que la incorporación de sus semillas en la dieta podría ayudar a aliviar, al menos en parte, el déficit en la ingesta de fibra (Alvarez-Jubete, Arendt y Gallagher, 2010). Además, posee un alto contenido de minerales, vitaminas y polifenoles (Navruz-Varli y Sanlier 2016).

En nuestro país, la barra de cereal se popularizó aproximadamente en el año 2000, cuando surgió como un producto destinado principalmente a deportistas, por su alto aporte de carbohidratos y proteínas, nutrientes que contribuyen a mejorar el rendimiento físico. Posteriormente, nacieron 
nuevas versiones destinadas a personas diabéticas, donde la cantidad de glúcidos se encuentra disminuida por el agregado de edulcorante artificial. El Código Alimentario Argentino (CAA) en el artículo 645, ítem 2, define a las barras de cereal como "Cereales aplastados, laminados, cilindrados o roleados (Rolled Cereals), preparados con granos limpios liberados de sus tegumentos y que después de calentados o de ligera torrefacción se laminan convenientemente". La barra de cereal sin cocción es el resultado de una mezcla de jarabe con o sin azúcar, cereal, frutas y/o semillas.

Debido a que el grano de quinoa posee un alto valor nutricional, una buena apariencia (color, textura, pequeño tamaño) y un sabor suave y agradable, su incorporación en barras de cereal sería una buena opción ya que es un producto de consumo masivo por la población general; y además facilitaría la incorporación de un cultivo andino a la alimentación diaria. Farinazzi-Machado, Barbalho, Oshiiwa, Goulart y Pessan Junior (2012), en su estudio determinan que el consumo de barras de cereal elaboradas con quinoa reduce los factores de riesgo relacionados con las enfermedades cardiovasculares, sin embargo, los autores no evaluaron la calidad de las barras elaboradas.

El objetivo del presente trabajo es estudiar el efecto de la sustitución de arroz crocante por semillas de quinoa sobre la calidad tecnológica, nutricional y sensorial de las barras de cereal.

\section{MATERIALES Y MÉTODOS}

\section{Materiales}

En la Tabla 1 se muestran los distintos ingredien- tes utilizados en la formulación de las barras de cereal. El arroz crocante (Marlenheim SA), los copos de maíz (Aro, Tres Arroyos SA), la avena arrollada (Aventres, Molino Sytari SRL), el arroz inflado (Negen, Cerexpa SRL) y la manzana parcialmente deshidratada (Banner, Gregorio Numo y Noel Werthein SA) y los demás aditivos (grado alimenticio) fueron provistos por la firma Georgalos SA ya que son los ingredientes utilizados en la empresa para la elaboración de barras de cereal. Las semillas de quinoa utilizadas fueron obtenidas en el mercado local, libres de saponinas, por lo que no fue necesario realizar ningún tratamiento previo a su uso.

\section{Elaboración de barras de cereal}

Las barras de cereal se elaboraron tomando como control a la fórmula de barra de cereal light de manzana de Georgalos SA. A partir de esta formulación control se elaboraron tres muestras de barras de cereal con diferentes porcentajes de semillas de quinoa (5, 10 y $15 \%$ ) sustituyendo el arroz crocante.En primer lugar, se preparó el jarabe donde el agua, la polidextrosa, el maltitol y la goma arábiga fueron cocinados durante 15 min a $116{ }^{\circ} \mathrm{C}$ hasta obtener un jarabe con $85^{\circ} \mathrm{Brix}$. El jarabe cocinado se llevó por medio de una bomba a un "tanque pesador", y se incorporó a la mezcladora tipo doble Z (Cariglino INCA. Buenos Aires, Argentina), posteriormente se agregó el sorbitol, esencia de manzana, aceite vegetal hidrogenado, cloruro de sodio y ácido cítrico. Luego se incorporó la quinoa (excepto para la muestra control), los copos de maíz, el arroz crocante, la manzana parcialmente deshidratada, la avena arrollada y el arroz inflado, y se mezclaron todos los ingredientes por 13 min hasta obtener una pasta uniforme.

Tabla 1. Formulaciones de las barras de cereal.

\begin{tabular}{lcccc}
\hline Ingredientes (\%p/p) & Control & $\mathbf{5 \%}$ Quinoa & $\mathbf{1 0} \%$ Quinoa & $\mathbf{1 5}$ Quinoa \\
\hline Quinoa & $\mathbf{0 , 0 0}$ & $\mathbf{5 , 1 0}$ & $\mathbf{1 0 , 1 9}$ & $\mathbf{1 5 , 2 9}$ \\
Arroz crocante & $\mathbf{1 8 , 2 2}$ & $\mathbf{1 3 , 1 2}$ & $\mathbf{8 , 0 3}$ & $\mathbf{2 , 9 3}$ \\
Copos de maíz & 18,22 & 18,22 & 18,22 & 18,22 \\
Manzana parcialmente deshidratada & 12,15 & 12,15 & 12,15 & 12,15 \\
Avena arrollada & 7,29 & 7,29 & 7,29 & 7,29 \\
Aceite vegetal hidrogenado & 3,28 & 3,28 & 3,28 & 3,28 \\
Arroz inflado & 0,98 & 0,98 & 0,98 & 0,98 \\
Maltitol & 19,65 & 19,65 & 19,65 & 19,65 \\
Polidextrosa & 18,52 & 18,52 & 18,52 & 18,52 \\
Goma arábiga (INS: 414) & 0,70 & 0,70 & 0,70 & 0,70 \\
Sorbitol (INS: 420) & 0,66 & 0,66 & 0,66 & 0,66 \\
Esencia de manzana & 0,11 & 0,11 & 0,11 & 0,11 \\
Ácido cítrico (INS: 330) & 0,11 & 0,11 & 0,11 & 0,11 \\
Cloruro de sodio & 0,11 & 0,11 & 0,11 & 0,11 \\
\hline
\end{tabular}


La pasta obtenida fue laminada pasando primero por un rolo prelaminador de $22 \mathrm{~mm}$ de apertura, y luego por un rolo laminador de $18 \mathrm{~mm}$ de apertura. Posteriormente esta lámina fue compactada y alisada, y se llevó a cabo el proceso de enfriamiento a través del paso por dos túneles de frío (uno antes del corte y otro posterior al corte) (SOGEM FL-TRB 800. Paimboeuf, Francia). Las pastas fueron cortadas en forma longitudinal por cuchillas múltiples que determinaron el ancho de la barra, y luego se realizó un corte en forma transversal por guillotina y se empacaron con una máquina tipo flow-pack (EUROSICMA-SEGRANTE. Milán, Italia). Todo el equipamiento usado en la elaboración de las barras de cereal se encontraba calibrado y certificado ante entidades homologadas, y se realizaron controles de acuerdo al procedimiento del Manual de Calidad Georgalos SA.

\section{Dimensiones y color de las barras de cereal}

Se determinaron las dimensiones de cada barra de cereal utilizando un calibre (Vernier Caliper Stronger, China) y se registró su peso en gramos. El color de las barras de cereal se determinó según el método 14-22 (AACC Internacional 2000) mediante un espectrofotómetro de reflectancia (Minolta 508d, Estados Unidos) de $8 \mathrm{~mm}$ de apertura, ángulo de observador $10^{\circ}$, iluminante D65, componente especular incluido. Los datos se recogieron en escala CIE-LAB ( $L^{*}$ : luminosidad; $a^{*}$ : tonalidad del rojo al verde; y $b^{*}$ : tonalidad del amarillo al azul).

\section{Dureza de las barras de cereal}

La dureza de las barras de cereal se evaluó mediante un texturómetro INSTRON (Universal Testing Machine, modelo 3342, Estados Unidos). Se realizó un test de compresión de acuerdo al método 74-09 (AACC Internacional 2000) mediante una sonda de 2,5 cm de diámetro bajo las siguientes condiciones de medición: celda de compresión de $500 \mathrm{~N}$, velocidad del cabezal 1,66 mm/seg; deformación $40 \%$. Se registraron los valores de fuerza requerida para comprimir la muestra hasta el $40 \%$ de su espesor original expresada como dureza $(\mathrm{N})$ (BluehillR 2.27 Instron).

\section{Composición centesimal de las barras de cereal}

Se determinó la composición centesimal de cada muestra mediante métodos aprobados de la
AACC Internacional (2000). Se determinó el contenido de humedad (44-19), el contenido de cenizas (08-01), proteínas (46-10), lípidos mediante una extracción en Soxhlet (30-25) y los carbohidratos totales se calcularon por diferencia. Cada determinación se expresó como porcentaje en base húmeda.

\section{Fibra alimentaria total}

Se determinó el contenido de fibra alimentaria total (FAT) de acuerdo al método 32-05 de la AACC Internacional (2000) a través del uso de un kit de ensayo (Megazyme International, Irlanda).

\section{Digestibilidad in vitro del almidón}

La digestión in vitro del almidón se realizó mediante el método multienzimático sin diálisis de acuerdo a la técnica de Bustos, Pérez y León (2011). A $4 \mathrm{~g}$ de cada muestra se les agregó $20 \mathrm{ml}$ de buffer fosfato salino $\mathrm{pH} \mathrm{6,9} \mathrm{(0,01} \mathrm{M} \mathrm{sales} \mathrm{fosfa-}$ to; $0,12 \mathrm{M} \mathrm{NaCl}$ y $2,7 \mathrm{~m} \mathrm{MKCl}$ ). Posteriormente, se ajustó el pH a 1,5 y se realizó una digestión con pepsina $(115 \mathrm{U} / \mathrm{ml})$ a $37^{\circ} \mathrm{C}$ por $30 \mathrm{~min}$. Luego, se reajustó el pH a 6,9 y se llevó a un volumen final de $50 \mathrm{ml}$ con buffer fosfato $\mathrm{pH}$ 6,9 y alfa-amilasa pancreática porcina $(110 \mathrm{U} / \mathrm{ml})$. Las muestras fueron incubadas por $3 \mathrm{~h}$ a $37^{\circ} \mathrm{C}$ y cada 30 min se tomaron alícuotas para realizar el análisis de azúcares reductores usando el método del ácido 3,5-dinitrosalicilico (DNS). Se utilizó una curva estándar de maltosa y un factor de conversión a almidón de 0,9. Se construyeron curvas de porcentaje de almidón hidrolizado vs. tiempo, las cuales fueron ajustadas a un modelo de primer orden según Goñi et al. (1997):

$$
\mathrm{C}=\mathrm{C}_{\infty}\left(1-\mathrm{e}^{-\mathrm{kt}}\right)
$$

donde $C$ corresponde al porcentaje de almidón hidrolizado en el tiempo $t ; C_{\infty}$ es el porcentaje de equilibrio de almidón hidrolizado a tiempo $\infty$; $K$ es la constante cinética y $t$ es el tiempo (min). La estimación de los parámetros se llevó a cabo usando el software ORIGIN PRO8.

Existe otra clasificación del almidón en función de su digestibilidad, definiéndose tres fracciones: almidón rápidamente digerible (ARD), aquel hidrolizado dentro de los primeros 20 min; almidón lentamente digerible (ALD), degradado entre los 20 y 120 min, y almidón resistente (AR) aquel hidrolizado luego de transcurridos 120 min (Englyst, K., Englyst H., Hudson, Cole y Cummings, 1999). A través de la utilización de las ecuaciones de ajuste a la curva de datos experimentales de la hidrólisis del almidón, se calculó la cantidad de ARD, ALD y 
de AR, teniendo en cuenta como base de almidón digerible $(\mathrm{AD})$ al correspondiente a $C_{\infty}$.

\section{Análisis sensorial}

Se llevó a cabo el análisis sensorial, a fin de evaluar la aceptabilidad global de barras de cereal con distintos porcentajes de semillas de quinoa. Para ello, se seleccionaron jueces no entrenados de la provincia de Córdoba, cuyo criterio de inclusión fue la ausencia de alergias alimentarias y/o de enfermedad de las vías respiratorias. El análisis sensorial se llevó a cabo con 57 jueces no entrenados tomados al azar: 38 mujeres y 19 hombres, cuyas edades fluctuaron entre 11 y 40 años.

Los parámetros evaluados fueron: color, olor, sabor, persistencia del mismo, textura, aspecto visual y aceptabilidad general de las barras de cereal. Se utilizó una escala hedónica de 9 puntos (1: Disgusta muchísimo; 9: Gusta muchísimo). También se les mencionó a los evaluadores una serie de atributos (crocancia, humedad, dureza, facilidad de masticar y pegajosidad), y se les pidió que indicaran si estas propiedades podrían ser asociadas a alguna de las muestras.

\section{Análisis estadístico}

El análisis estadístico de los resultados se realizó mediante el software estadístico InfoStat (Di Rienzo et al, 2011). Los ensayos se realizaron al menos por duplicado y se informaron las medias con sus desviaciones estándar. Los resultados obtenidos fueron evaluados estadísticamente mediante análisis de varianza (ANOVA). En los casos que se presentaron diferencias, la comparación de las medias se realizó mediante el test de Fisher (LSD) con un nivel de significancia de 0,05.

\section{RESULTADOSY DISCUSIÓN}

\section{Calidad tecnológica de las barras de cereal con quinoa}

Las barras de cereal enriquecidas con quinoa presentaron mayor peso al incrementar el porcen- taje de quinoa respecto al control (Tabla 2). Este incremento se debió a que la semilla ocupa menos volumen que el arroz crocante, por lo que, con las dimensiones ya establecidas por el equipamiento usado en la elaboración, permite incorporar más cantidad de mezcla por unidad de volumen. Por otra parte, se observó una disminución significativa en el largo de las barras de cereal a medida que se incrementó el contenido de quinoa $(p<0,05)$, siendo la barra control la de mayor longitud. Tal como se explicó anteriormente, las diferencias en el tamaño entre las semillas de quinoa y el arroz crocante probablemente sea la causa de las variaciones en las dimensiones entre las muestras (Figura 1) ya que son fuertemente afectados por cambios en las formulaciones. Además, los cambios observados respecto al tamaño pueden deberse a una contracción de las barras que se produce posteriormente al corte, dado el paso por el segundo túnel de frío. Un aspecto importante que afecta la aceptabilidad del producto es el color de la superficie de las barras de cereal. La sustitución de arroz crocante por quinoa provocó cambios en los valores de $L^{*}$ (luminosidad) y $a^{*}$ (tonalidad del verde-rojo) sin modificar significativamente la tonalidad amarilla $\left(b^{*}\right)$ (Tabla 2). La mayor tonalidad rojiza (valores de $a^{*}$ más altos) en las barras de cereal al aumentar el porcentaje de quinoa en la formulación $(p<0,05)$ puede explicarse por el pequeño tamaño de las

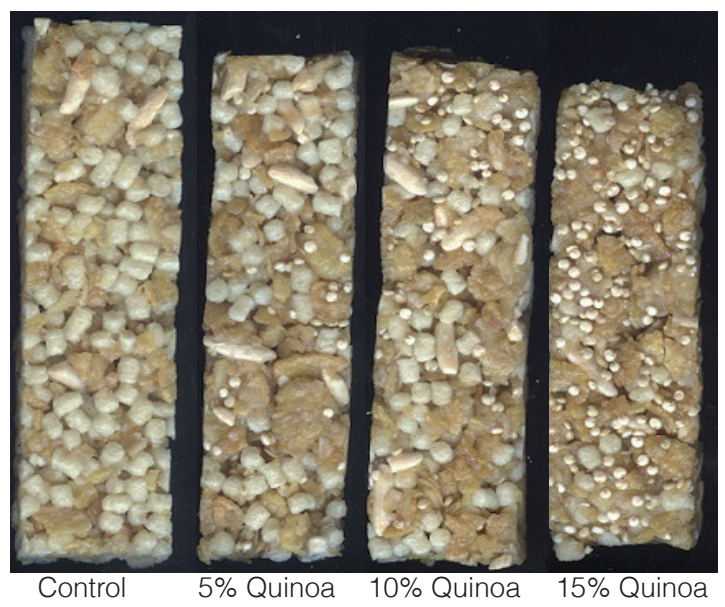

Figura 1. Barras de cereal.

Tabla 2. Parámetros de calidad tecnológica de las barras de cereal.

\begin{tabular}{lcccccc}
\hline Barras & PESO (g) & LARGO (mm) & Dureza (N) & $\mathbf{L}^{*}$ & $\mathbf{a}^{*}$ & $\mathbf{b}^{\mathbf{*}}$ \\
\hline Control & $23,01 \mathrm{a}$ & $107,3 \mathrm{~b}$ & $216,9 \mathrm{c}$ & $67,83 \mathrm{~b}$ & $3,92 \mathrm{a}$ & $28,87 \mathrm{a}$ \\
$\mathbf{5} \%$ Quinoa & $29,01 \mathrm{~b}$ & $102,9 \mathrm{a}$ & $150,9 \mathrm{~b}$ & $65,76 \mathrm{~b}$ & $6,65 \mathrm{ab}$ & $31,55 \mathrm{a}$ \\
$\mathbf{1 0} \%$ Quinoa & $29,05 \mathrm{~b}$ & $103,9 \mathrm{a}$ & $139,2 \mathrm{~b}$ & $61,48 \mathrm{a}$ & $9,74 \mathrm{~b}$ & $30,56 \mathrm{a}$ \\
$\mathbf{1 5} \%$ Quinoa & $31,98 \mathrm{~b}$ & $103,7 \mathrm{a}$ & $112,4 \mathrm{a}$ & $62,46 \mathrm{a}$ & $9,54 \mathrm{~b}$ & $30,28 \mathrm{a}$ \\
\hline
\end{tabular}


semillas de quinoa que genera que la tonalidad de los copos de maíz se destaque (Figura 1). Además, también hay que considerar el diferente color de la quinoa con respecto al arroz crocante (más blanquecino), que puede haber causado una menor luminosidad en la superficie de las barras de cereal. Es importante aclarar, que el color en la superficie de las muestras es heterogéneo debido a la diversidad de componentes utilizados en su formulación. Sin embargo, esta misma propiedad resulta atractiva para los consumidores porque refleja la presencia de distintos cereales y frutas que la componen. Las barras de cereal con quinoa presentaron una menor dureza, a medida que aumentó el porcentaje de sustitución $(p<0,05)$ (Tabla 2$)$. La textura de los alimentos depende de la microestructura de éstos y de las fuerzas físicas que hay entre los distintos componentes (Stanley, 1986). El reemplazo del arroz crocante por las semillas de quinoa produce un cambio en la estructura interna de las barras debilitando las interacciones entre los componentes, lo que se refleja en una menor dureza. Por otro lado, en el proceso de elaboración del arroz crocante se produce una gelatinización del almidón que luego durante el almacenamiento retrograda, mientras que en las semillas de quinoa el almidón se encuentra sin gelatinizar empaquetado dentro de los gránulos. Una mayor retrogradación del almidón de algunos componentes de las barras podría conducir a una mayor dureza de éstas, como es el caso de la barra control (Wang, Li, Copeland, Niu y Wang, 2015).

\section{Calidad nutricional de las barras de cereal con quinoa}

El refinamiento de los granos consiste en un proceso que genera la pérdida de vitaminas, minerales y fibra alimentaria total. Una de las estrategias sugeridas consiste en la sustitución de granos refinados por granos enteros. Diversos estudios han demostrado que el consumo de granos de cereales intactos se encuentra directamente relacionado con el mantenimiento de una buena salud y el control de enfermedades relacionadas con la dieta, tales como enfermedades cardiovasculares, diabetes tipo 2 y algunos tipos de cáncer (Jacobs, Marquart, Slavin y Kushi,1998; Meyer et al., 2000; Kasum, Jacobs, Nicodemus y Folsom, 2002). En este sentido, la sustitución de arroz crocante en la formulación de las barras de cereal por granos enteros de quinoa podría causar una mejora en el perfil nutricional. La composición de las distintas barras de cereal se presenta en la Tabla 3. No se observaron diferencias significativas en el contenido de humedad por el agregado de quinoa en la formulación, obteniéndose un valor promedio de $10 \%$, valor reconocido como adecuado para la conservación de este tipo de productos, ya que no permite el crecimiento microbiano y fúngico. Tampoco se observaron diferencias significativas en el contenido de carbohidratos. Con respecto al contenido de minerales, se observó un leve aumento $(p<0,05)$, a medida que se incrementó la cantidad de quinoa en la formulación de las barras de cereal; esto podría deberse, por un lado, a que la semilla de quinoa no se encuentra refinada $y$, por otro lado, a que posee un mayor contenido de minerales con respecto al arroz. Los principales minerales de la quinoa son el potasio, el hierro, el calcio, el zinc y el magnesio, y en general se encuentran en mayor proporción que en el resto de los cereales (Nowaky Charrondiere, 2015). El contenido de proteína total de la quinoa es mayor que la del arroz y como consecuencia del reemplazo hubo un incremento del contenido de proteínas de las barras (Tabla 3). Las proteínas de la quinoa son ricas en lisina (aminoácido limitante en la mayoría de los cereales), y su biodisponibilidad no se vería afectada en las barras de cereal porque no sufrieron un tratamiento térmico durante su elaboración. Además, la quinoa posee todos los aminoácidos esenciales (Abougoh et al., 2008; Steffolani et al., 2015) y de acuerdo a las recomendaciones de la WHO (1985), algunas variedades de quinoa no poseen aminoácidos limitantes para niños en edad escolar por lo que podría ser una alternativa de colación en los comedores escolares. Los valores de fibra alimentaria total (FAT) de las barras de cereal no incluyeron en su porcentaje a la polidextrosa, ya que no puede determinarse mediante la metodología disponible

Tabla 3. Composición de las barras de cereal.

\begin{tabular}{lcccccc}
\hline Barras & $\begin{array}{c}\text { Humedad } \\
\text { (\%, bh) }\end{array}$ & $\begin{array}{c}\text { Cenizas } \\
\mathbf{( \% ,}, \mathbf{b h})\end{array}$ & $\begin{array}{c}\text { Lípidos } \\
\mathbf{( \% ,}, \mathbf{b h})\end{array}$ & $\begin{array}{c}\text { Proteínas } \\
\text { (\%, bh) }\end{array}$ & $\begin{array}{c}\text { Carbohidratos } \\
\text { (\%, bh) }\end{array}$ & $\begin{array}{c}\text { FAT* } \\
\text { (\%, bh) }\end{array}$ \\
\hline Control & $10,17 \mathrm{a}$ & $0,76 \mathrm{a}$ & $8,09 \mathrm{c}$ & $4,15 \mathrm{a}$ & $76,85 \mathrm{a}$ & $4,1 \mathrm{a}$ \\
$\mathbf{5} \%$ Quinoa & $10,75 \mathrm{a}$ & $0,98 \mathrm{~b}$ & $7,39 \mathrm{~b}$ & $3,94 \mathrm{a}$ & $77,94 \mathrm{ab}$ & $5,1 \mathrm{ab}$ \\
$\mathbf{1 0} \%$ Quinoa & $10,54 \mathrm{a}$ & $1,09 \mathrm{c}$ & $7,38 \mathrm{~b}$ & $4,79 \mathrm{ab}$ & $76,20 \mathrm{a}$ & $6,8 \mathrm{bc}$ \\
$\mathbf{1 5 \%}$ Quinoa & $10,15 \mathrm{a}$ & $1,08 \mathrm{c}$ & $6,39 \mathrm{a}$ & $5,39 \mathrm{c}$ & $76,00 \mathrm{a}$ & $6,9 \mathrm{c}$ \\
\hline
\end{tabular}

etras diferentes en cada columna indican diferencias significativas con $\mathrm{p}<0,05$

*FAT: Fibra Alimentaria Total 
en el presente estudio. Sin embargo, el porcentaje de polidextrosa utilizado en todas las muestras fue el mismo, incluido el control, por lo tanto, los valores informados nos permiten comparar si hay o no un incremento en el contenido de fibra al incorporar quinoa. En la Tabla 3, se muestran los valores obtenidos de FAT, donde se observó un aumento con el incremento del contenido de quinoa en la formulación de las barras de cereal. Este incremento se debió principalmente a que, a diferencia del arroz crocante, la quinoa se encuentra como grano entero, por lo tanto, posee un alto porcentaje de fibra. Las barras de cereal con diferente proporción de quinoa presentaron los siguientes incrementos: la muestra con el $5 \%$ tiene un $24,4 \%$ de aumento en fibra con respecto al control; la del $10 \%$ un $65,9 \%$; y la del $15 \%$ supera en un $68,3 \%$ a la de control. A partir de estos porcentajes obtenidos, se le puede acreditar a las barras con el 10 y $15 \%$ de quinoa, el atributo "Aumentado en fibras" de acuerdo al CAA.

\section{Digestibilidad in vitro del almidón}

La digestibilidad in vitro del almidón de las barras de cereal fue seguida mediante el monitoreo de la cantidad de azúcares reductores liberados en el proceso. Estos resultados son un buen indicador de los diferentes parámetros de degradación del almidón imitando algunas condiciones del

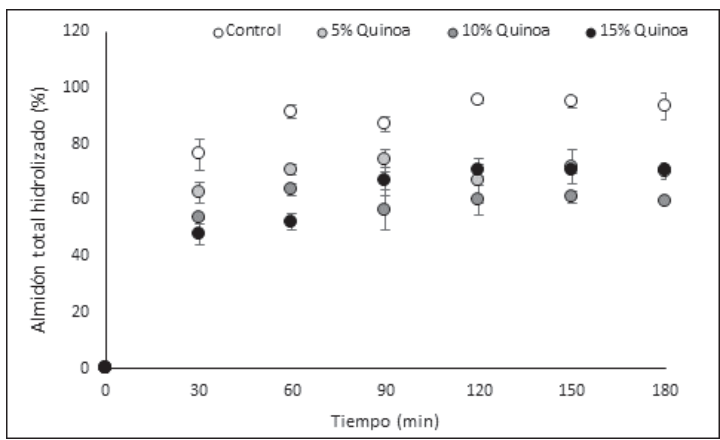

Figura 2. Curva de hidrólisis de almidón de las barras de cereal. Control (blanco), barra con $5 \%$ de quinoa (gris claro), barra con $10 \%$ de quinoa (gris oscuro) y barra con $15 \%$ de quinoa (negro). sistema gastrointestinal. Las curvas de hidrólisis de almidón obtenidas para cada muestra (Figura 2) presentaron un buen ajuste a la ecuación $\mathrm{C}=\mathrm{C}_{\infty}$ $\left(1-e^{-K t}\right)$ con un $R^{2}$ por encima de 0,97 en todos los casos, lo que demuestra que el modelo utilizado describe los datos obtenidos de forma adecuada. A partir de las curvas ajustadas a esta ecuación, se calculó el porcentaje de hidrólisis del almidón a tiempo infinito $\left(C_{\infty}\right)$ y la constante cinética $(K)$ (Tabla 4).

Las muestras enriquecidas con semillas de quinoa presentaron una menor velocidad de hidrólisis (pendiente menor), comparado con el control, indicando una menor liberación de azúcares reductores (principalmente maltosa) a partir de la digestión más lenta del almidón. Sin embargo, no se observaron importantes diferencias entre los distintos niveles de sustitución. Es importante destacar que durante los primeros 30 min las muestras con incorporación de quinoa presentaron valores de digestibilidad de almidón inversamente proporcional al porcentaje de sustitución. Este resultado es muy importante debido que la mayor proporción de glucosa se libera en este período como resultado de la hidrólisis de la fracción de almidón rápidamente digerible (Gelencsér, Gál, Hódságiand y Salgó, 2007). Esta disminución de la velocidad de hidrólisis con incorporación de semillas de quinoa, convierte a las barras de cereal enriquecidas en un producto apropiado para personas con problemas de salud relacionados al metabolismo de la glucosa (Willett, Manson y Liu, 2002). A mayores tiempos de digestión, la velocidad de hidrólisis comienza a disminuir hasta estabilizarse en un valor determinado $\left(C_{\infty}\right)$, el cual fue significativamente menor cuando en la formulación de las barras se incorporó quinoa. En este sentido, el menor porcentaje de almidón hidrolizado al finalizar la digestión fue observado en la muestra con $10 \%$ de semillas de quinoa (Tabla 4). Esto indica que las muestras enriquecidas podrían presentar un menor índice glucémico.

La cinética de la digestión del almidón puede ser afectada por varios factores, como la naturaleza del almidón (Ao et al., 2007), la presencia de

Tabla 4. Parámetros de digestibilidad in vitro del almidón de las barras de cereal.

\begin{tabular}{|c|c|c|c|c|c|}
\hline Barras & $\begin{array}{l}{ }^{1} \mathrm{C}_{\infty} \\
(\%)\end{array}$ & $\begin{array}{c}{ }^{2} \mathrm{~K} \\
(\min -1)\end{array}$ & $\begin{array}{c}{ }^{3} \mathrm{ARD} \\
\left(\mathrm{g} / 100 \mathrm{de}^{6} \mathrm{AD}\right)\end{array}$ & $\begin{array}{c}{ }^{4} \mathrm{ALD} \\
\left(\mathrm{g} / 100 \mathrm{de}{ }^{6} \mathrm{AD}\right)\end{array}$ & $\begin{array}{c}{ }^{5} \mathrm{AR} \\
\left(\mathrm{g} / 100 \mathrm{de}{ }^{6} \mathrm{AD}\right)\end{array}$ \\
\hline Control & $94 \mathrm{c}$ & $0,05 a$ & $63 c$ & $32 \mathrm{c}$ & $0,13 b$ \\
\hline 5\% Quinoa & $69 b$ & $0,11 \mathrm{c}$ & $60 c$ & $9 a$ & $0,00 a$ \\
\hline $10 \%$ Quinoa & $60 a$ & $0,08 b$ & $47 b$ & $13 b$ & $0,01 a$ \\
\hline $15 \%$ Quinoa & $71 b$ & $0,04 a$ & $41 a$ & $29 c$ & $0,33 c$ \\
\hline
\end{tabular}

Letras diferentes en cada columna indican diferencias significativas con $\mathrm{p}<0,05$.

${ }^{1}$ Concentración de equilibrio $(\mathrm{C} \infty),{ }^{2}$ Constante cinética $(\mathrm{K}),{ }^{3} \mathrm{Almidón} \mathrm{rápidamente} \mathrm{digerible} \mathrm{(ARD),}{ }^{4}$ Almidón lentamente digerible (ALD), ${ }^{5}$ Almidón resistente (AR), ${ }^{6}$ Almidón digerible (AD) 
fibra y antinutrientes (Brennan, Blake, Ellis y Schofield, 1996) y los cambios de las interacciones que tienen lugar durante el procesamiento, por lo que el almidón presente en los alimentos no es completamente digerido (Singh, Dartois y Kaur, 2010). Probablemente, el menor porcentaje de hidrólisis del almidón de las barras con quinoa se deba a que el almidón del arroz crocante que se encuentra gelatinizado (como se mencionó anteriormente) está más expuesto al ataque de las enzimas del tracto intestinal, mientras que el almidón de los granos de quinoa se encuentra empaquetado dentro de los gránulos, quedando menos disponible a la acción enzimática.

En la Tabla 4 es posible apreciar, tal como se observó anteriormente, que la barra de cereal control presentó el mayor contenido de ARD, lo cual se corresponde con la mayor magnitud de hidrólisis observada $\left(\mathrm{C}_{\infty}\right)$. Las muestras enriquecidas con quinoa mostraron una disminución de dicha fracción de almidón $\left(\mathrm{C}_{\infty}\right)$ con el aumento del porcentaje de quinoa incorporada $(p<0,05)$ de acuerdo a los resultados descriptos. Una mayor constante cinética de hidrólisis de almidón lleva a que en los primeros minutos de digestión una gran cantidad del mismo sea hidrolizado disminuyendo las fracciones de ALD y AR. En este sentido, la fracción de ALD fue aumentando conforme disminuyó la constante cinética $(K)$ y a medida que se incrementó el porcentaje de sustitución de quinoa. En la fracción de almidón que se considera resistente en estas condiciones de ensayo se observó la misma tendencia, siendo la muestra con el máximo nivel de sustitución la que presentó el máximo valor (Tabla 4). Sin embargo, estos valores de almidón resistente son despreciables en relación al almidón total digerible. Estos resultados demuestran que las diferencias observadas en la digestibilidad de almidón se deben principalmente a una disminución de la fracción de almidón rápidamente digerible. Los cambios en la cinética de digestión del almidón pueden ser debidos a que, al incorporar quinoa en la formulación, la naturaleza del almidón cambia y, además, durante el procesamiento y almacenamiento, la amilosa principalmente puede formar complejos con otros macronutrientes volviéndose menos susceptible al ataque de enzimas amilolíticas.

\section{Análisis sensorial de las barras de cereal con quinoa}

En la Figura 3 se muestran los resultados obtenidos en la evaluación del sabor, de la textura, el aspecto visual y la aceptabilidad general de las barras de cereal. Un mayor número de jueces evaluó el sabor de las barras con quinoa con un puntaje de 7 (gusta mucho), mientras que a la barra control, el mayor número de jueces la evaluó con un puntaje inferior (6: gusta ligeramente). En la puntuación promedio general, los evaluadores no percibieron diferencias respecto a los atributos color y olor; el $50 \%$ de los jueces calificó a todas las barras de cereal por encima de 6 (gusta ligeramente) al atributo color, y por encima de 5 (ni disgusta, ni gusta) al atributo olor (datos no mostrados). Alrededor del $30 \%$ de los jueces calificó a la textura de las barras de cereal enriquecidas con quinoa con 6 (gusta ligeramente) y 7 (gusta mucho), mientras que el control tuvo bajos porcentajes de jueces que calificaron de forma agradable su textura y el mayor porcentaje de jueces la calificó con 4 (disgusta ligeramente) (Figura 3B). La muestra control fue asociada a la característica "dura" por el $40 \%$ de los evaluadores, lo cual coincide con la dureza evaluada instrumentalmente, explicando la preferencia observada para las muestras con quinoa. En la evaluación del aspecto visual de las barras de cereal, se observó que el $15 \%$ de sustitución de arroz crocante por semillas de quinoa generó un cambio importante en la aceptabilidad con un aumento de calificaciones positivas, lo cual indicaría la buena predisposición de los consumidores a adquirir productos con la incorporación de cereales o semillas no tradicionales (Figura 3C). La aceptabilidad general de las barras de cereales enriquecidas con semillas de quinoa aumentó notablemente respecto del control con calificaciones de 7 (gusta mucho) y 8 (gusta bastante). El mayor porcentaje de jueces evaluó a la barra control y a las barras con $5 \%$ de quinoa con la calificación de 6 (gusta ligeramente), y a la barra con 10 y $15 \%$ de quinoa con 7 (gusta mucho) (Figura 3D). Al aumentar el porcentaje de quinoa incorporado en la formulación de barras de cereal, las muestras resultaron menos crocantes y menos duras, como así también, más húmedas, fáciles de masticar y pegajosas respecto del control, lo que explica por qué aquellas muestras con 10 y $15 \%$ de semillas de quinoa resultaron ser las más aceptadas por los jueces.

\section{CONCLUSIÓN}

La diferencia de tamaño entre el arroz crocante y las semillas de quinoa provocó modificaciones en el peso y en las dimensiones del producto final, resultando las barras con quinoa con un peso mayor y más cortas. Además, las barras de cereal enriquecidas con quinoa fueron más blandas y levemente más oscuras; estos cambios se 

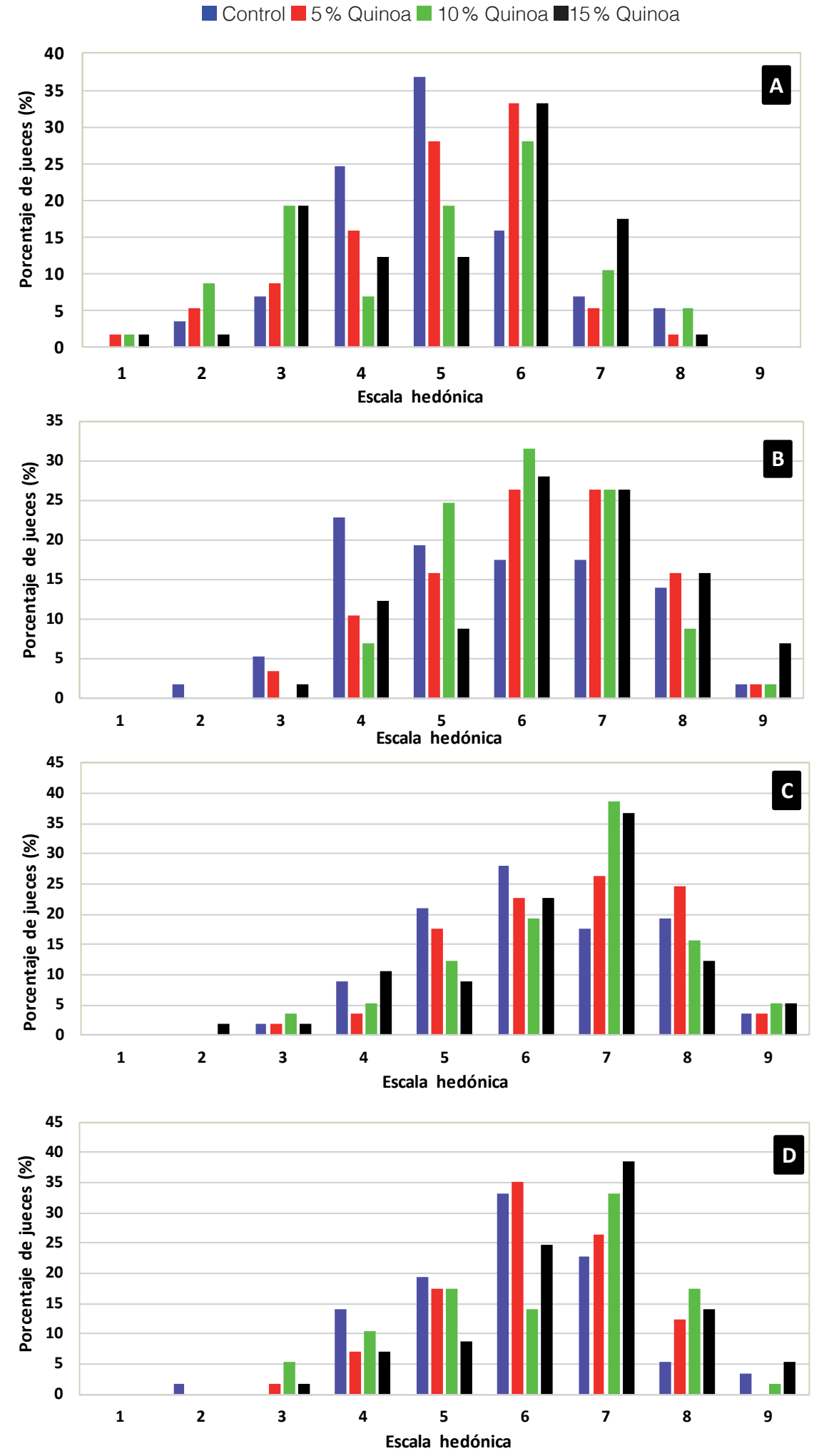

Figura 3. Valoración del sabor, la textura, el aspecto visual y la aceptabilidad general en la evaluación sensorial de barras de cereal enriquecidas con quinoa (A): Sabor; (B): Textura; (C): Aspecto visual; (D): Aceptabilidad general. 
debieron principalmente a que el pequeño tamaño de las semillas de quinoa generó que la tonalidad de los copos de maíz se destaque. Por otro lado, el reemplazo del arroz crocante por las semillas de quinoa causó un cambio en la estructura interna de las barras debilitando las interacciones entre sus componentes, lo que se reflejó en una menor dureza. La incorporación de quinoa en la formulación permitió incrementar el contenido de proteínas y minerales, y el porcentaje de fibra que presentaron las barras con $10 \%$ y $15 \%$ de quinoa les permitió la clasificación como "Aumentado en fibra" de acuerdo al CAA. Los ensayos de digestibilidad in vitro del almidón mostraron que las barras con quinoa presentaron una significativa reducción en la liberación de maltosa, debido a una disminución de la fracción de almidón rápidamente digerible lo cual las hace más apropiadas para personas con problemas en el metabolismo de la glucosa. En el análisis sensorial, se observó que la incorporación de semillas de quinoa aumentó la aceptabilidad general de las barras enriquecidas con respecto al control, aceptabilidad fuertemente vinculada a la asociación de las barras de cereales con quinoa con productos menos crocantes y duros, más húmedos y de fácil masticación.

\section{AGRADECIMIENTOS}

Los autores agradecen a la empresa Georgalos Hnos. SAICA por prestar las instalaciones para elaborar las barras de cereal, y a la Secretaría de Ciencia y Técnica de la Universidad Nacional de Córdoba por el apoyo económico recibido.

\section{BIBLIOGRAFÍA}

Abugoch, L., Romero, N., Tapia, C., Silva, J. y Rivera, M. (2008). Study of some physicochemical and functional properties of quinoa (Chenopodium quinoa Willd) protein isolates. Journal of Agricultural of Food Chemistry 56(12), 4745-4750.

Alvarez-Jubete, L., Arendt, E. K., y Gallagher, E. (2010). Nutritive value and chemical composition of pseudocereals as gluten-free ingredients. International Journal of Food Science and Nutrition 4, 240-257.

Ao, Z., Simsek, S., Zhang, G., Venkatachalam, M., Reuhsand, B. L., Hamaker, B. R. (2007). Starch with a slow digestion property produced by altering its chain length, branch density and crystaline structure. Journal of Agricultural Food Chemistry 55 (11), 45404547.

Brennan, C. S., Blake, D. E., Ellis, P. R. y Schofield, J. D. (1996). Effect of guar galactomannan on wheat bread microstructure and on the in vitro and in vivo digestibility of starch in bread. Journal of Cereal Science, 24, 151-160.

Bustos, M. C., Pérez, G. T. y León, A. E (2011). Sensory and nutritional attributes of fibre enriched pasta. LWT - Food Science and Technology 44, (6,), 1429-1434.

Di Rienzo, J. A., Casanoves, F., Balzarini, M. G., Gonzalez, L., Tablada, M., y Robledo, Y. C. (2011). InfoStat versión 2011. Grupo InfoStat, FCA, Universidad Nacional de Córdoba, Argentina. URL http://www. infostat. com. ar

Englyst, K. N., Englyst, H. N., Hudson, G. J., Cole, T. J.y Cummings, J. H. (1999). Rapidly available glucose in foods: an in vitro measurement that reflects the glycemic response. The American Journal of Clinical Nutrition, 69, 448-454.

WHO (1985). Energy and Protein Requirements. Report of a joint FAO/WHO/UNU expert consultation. WHO Technical Report, Series, № 724.

Farinazzi-Machado, F. M. V., Barbalho, S. M., Oshiiwa, M., Goulart, R. y Pessan Junior, O. (2012). Use of cereal bars with quinoa (Chenopodium quinoa W.) to reduce risk factors related to cardiovascular diseases. Ciência e Tecnologia de Alimentos 32(2), 239-244.

Gelencsér, T., Gál, V., Hódságiand, M. y Salgó, A. (2007). Evaluation of quality and digestibility characteristics of resistant starch-enriched pasta. Food Bioprocess Technology 1 (2), 171-179.

Jacobsen, S. E., Mujica, A. y Jensen, C. R. (2003). The resistance of quinoa (Chenopodium quinoa Willd) to adverse abiotic factors. Food Reviews International 19 (1-2), 99-109.

Jacobs, D. R., Marquart, L., Slavin, J. y Kushi, L. H. (1998). Whole-grain intake and cancer: an expanded review and meta-analysis. Nutrition and Cancer 30 (2), 85-96.

Kasum, C. M., Jacobs, D. R., Nicodemus, K. y Folsom, A. R. (2002). Dietary risk factors for upper aerodigestive tract cancers. International Journal of Cancer 99(2), 267-272.

LEY No 18284, Código Alimentario Argentino, Capítulo V, artículo 235 quinto. Resolución GMC № 01/02 (incorporada por Resolución Conjunta SPRel № 161/2013 y SAGyP № 213/2013), Reglamento técnico Mercosur sobre "Información nutricional complementaria (declaraciones de propiedades nutricionales)". Apartado 5 "Condiciones para declarar información nutricional complementaria (declaraciones de propiedades nutricionales)". Subapartado 5.2 "Contenido comparativo". Recuperado de: http://www.alimentosargentinos.gob. ar/HomeAlimentos/Marco_Regulatorio/ultimas\%20 modificaciones/Capitulo_V.pdf

Meyer, K. A., Kushi, L. H., Jacobs, D. R., Slavin, J. R., Sellers, T. A. y Folsom, A. R. (2000). Carbohydrates, 
dietary fiber, and incident type 2 diabetes in older women. The American Journal of Clinical Nutrition 71(4), 921-30.

Navruz-Varli, S. y Sanlier, N. (2016). Nutritional and health benefits of quinoa (Chenopodium quinoa Willd). Journal of Cereal Science 69,371-376.

Nowak, V., Du, J. U. y Charrondière, R. (2015). Assessment of the nutritional composition of quinoa (Chenopodium quinoa Willd). Food Chemistry 193, 47-54.

Stanley, D. W. (1986). Chemical and structural determinants of texture of fabricated foods. Food Technology, 12, 65-76.

Repo-Carrasco, R., Cortez, R. O. M., Quispe, V. y Ramos, I. (2006). "Cultivos Andinos", en León, E. y Rosell, M. C. De tales harinas, tales panes. Granos, harinas y productos de panificación Iberoamericana (pp. 245294). Córdoba, Argentina. Editorial ISEKI-Food.

Singh, J., Dartois, A. y Kaur, L. (2010). Starch digestibility in food matrix: a review. Trends in Food Science and Technology 21, 168-180.

Steffolani, M. E., Leon, A. E. y Perez, G. T. (2013). Study of the physicochemical and functional characterization of quinoa and kañiwastarches. Starch/Stärke 65(1112), 976-983.

Steffolani, M. E., Villacorta, P., Morales-Soriano, E. R., Repo-Carrasco, R., León, A. E. y Pérez, G. T. (2016). Physicochemical and Functional Characterization of Protein Isolated from Different Quinoa Varieties (Chenopodiumquinoa Willd). Cereal Chemistry 93(3), 275281.

Vega-Gálvez, A., Miranda, M. y Vergara, J. (2010). Nutrition facts and functional potential of quinoa (Chenopodium quinoa Willd), an ancient Andean grain: a review. Journal of the Science of Food and Agriculture 90(15), 2541-2547.

Wang Shujun, Li Caili, Copeland Les, Niu Qing y Wang Shuo (2015). Starch Retrogradation: A Comprehensive Review. Food Science and Food Safety 14 (5), 568585.

Willett, W., Manson, J. y Liu, S. (2002). Glycemic index, glycemic load and rick of tipe 2 diabetes. American Journal of ClinicalNutrition 76(1), 274-280. 